\title{
Cyber-Risk Identification for a Digital Substation
}

\author{
Athar Khodabakhsh \\ Norwegian University of Science and \\ Technology, NTNU \\ Gjøvik, Norway \\ athar.khodabakhsh@ntnu.no
}

\author{
Sule Yildirim Yayilgan \\ Norwegian University of Science and \\ Technology, NTNU \\ Gjøvik, Norway \\ sule.yildirim@ntnu.no
}

\author{
Mohamed Abomhara \\ Norwegian University of Science and \\ Technology, NTNU \\ Gjøvik, Norway \\ mohamed.abomhara@ntnu.no
}

\author{
Maren Istad \\ SINTEF Energy Research \\ Trondheim, Norway \\ maren.istad@sintef.no
}

\author{
Nargis Hurzuk \\ Statnett \\ Oslo, Norway \\ nargis.hurzuk@statnett.no
}

\begin{abstract}
Power grids are rapidly evolving through digital transformation by adopting information and communication technologies. This evolution is creating concerns for electricity transmission and distribution companies to maintain security and reliability of the power grid. To safeguard the power grids, cyber-security risks and their consequences on security measures are required to be analyzed. This paper applies cyber-risk identification methodology with focus on digital substation for recognizing potential cyber-attacks, evaluates the cyber-risks and their impacts, and defines mitigation plans to ensure reliable operation of digital substations and safe and secure delivery of reliable power.
\end{abstract}

\section{CCS CONCEPTS}

- Security and privacy $\rightarrow$ Network security.

\section{KEYWORDS}

cyber-attack, cyber-risk identification, digital substation, cybersecurity, IED, process bus, IEC 61850

\section{ACM Reference Format:}

Athar Khodabakhsh, Sule Yildirim Yayilgan, Mohamed Abomhara, Maren Istad, and Nargis Hurzuk. 2020. Cyber-Risk Identification for a Digital Substation. In The 15th International Conference on Availability, Reliability and Security (ARES 2020), August 25-28, 2020, Virtual Event, Ireland. ACM, New York, NY, USA, 7 pages. https://doi.org/10.1145/3407023.3409227

\section{INTRODUCTION}

Integration of information and communication technologies into industrial control system (ICS) through digitalization and automation are raising concerns about cyber-risks [Abomhara and Køien 2014; Knowles et al. 2015]. ICS automation aims to design and configure the industrial system in order to 1) provide real-time activity monitoring of assets across ICS including communication

Permission to make digital or hard copies of all or part of this work for personal or classroom use is granted without fee provided that copies are not made or distributed for profit or commercial advantage and that copies bear this notice and the full citation on the first page. Copyrights for components of this work owned by others than ACM must be honored. Abstracting with credit is permitted. To copy otherwise, or republish, to post on servers or to redistribute to lists, requires prior specific permission and/or a fee. Request permissions from permissions@acm.org.

ARES 2020, August 25-28, 2020, Virtual Event, Ireland

(C) 2020 Association for Computing Machinery.

ACM ISBN 978-1-4503-8833-7/20/08 . \$ 15.00

https://doi.org/10.1145/3407023.3409227 networks and applications, 2) provide real-time management of critical activities and configuration across the whole system, 3) provide defense against unauthorized access and use of the system. ICS consist of several assets such as supervisory control systems and data acquisition (SCADA), distributed control systems (DCS), and human machine interfaces (HMI) that are commonly used for monitoring and control of their critical infrastructure [Knowles et al. 2015]. To ensure reliable operation of the system, potential cyber-security risks on assets are required to be identified for appropriate countermeasure [Stouffer et al. 2011]. It is desirable to evaluate power system's vulnerability with respect to cyber-attack vectors and discover threat in order to apply appropriate mitigation plans.

A digital substation (DS) is a substation where most of hardwired copper connections are replaced by optical fiber cables and electronic devices from different vendors are integrated into power utilities control system [ECODIS 2019]. A substation takes the electrical energy from transmission network and decreases the voltage level by transformers. Moreover, DS's components communicate based on IEC 61850 global standard "Communication Networks and Systems in Substations" [Baigent et al. 2004]. Adaptation of IEC 61850 standard have enhanced reliability of substation operations, simplified engineering, and reduced costs. However, in the absence of solid security in place, malfunctions, and cyberattacks may result in security breaches in DS and outweigh DS benefits. DS development is rather new in Norway [Loken et al. 2018] and in ECODIS ${ }^{1} \mathrm{R} \& \mathrm{D}$ project, Statnett ${ }^{2}$ is investigating new functionality advantages and associated risks with IEC 61850 process bus technology. The ECODIS project seeks to utilize digital measurements to implement additional functionalities in real-time and investigate interoperability aspects of IEC 61850 to increase competence in transmission and distribution of power operators in Norway [ECODIS 2019]. Cyber-security related findings lay the foundation for implementing real-time functionalities for condition monitoring and facilitate an effective and secure deployment of DS [Stensrud et al. 2018].

In this paper, a 3-step methodology is applied for cyber-risk identification [MITRE 2020b] for ECODIS project. First, specific

\footnotetext{
${ }^{1}$ Engineering and Condition monitoring in Digital Substation (ECODIS) is a project funded by Research Council of Norway (NFR), Innovation Project for the Industrial Sector - ENERGIX program, project number 296550, coordinated by Statnett R\&D group.

${ }^{2}$ Statnett is the system operator of the Norwegian power system.
} 
cyber-attack vectors for DS are recognized. Second, tactics to affect the DS components are addressed by mapping the attacks to confidentiality, integrity, and availability (CIA) [Abomhara et al. 2015; Saltzer and Schroeder 1975]. The most relevant cyber-attack techniques (MITRE [MITRE 2020a]) that are possible through cyberattack vectors are categorized for each asset and their impact with respect to CIA. Third, mitigation mechanisms are addressed to ensure DS's stable operation and mitigate cyber-security risks to control systems [Moreira et al. 2016].

The main focus of this paper is on cyber-risk identification and evaluation of cyber-attack's impact on a DS. Section 2 describes assets in DS and their advanced functionalities. Section 3 describes the 3-step cyber-risks identification methodology through discovering cyber-attack vectors on DS, evaluating cyber-risks impacts mapped to identified vectors with respect to MITRE metrics, and defining mitigation plans in order to preserve CIA measures. Section 4 concludes the study and highlights future research.

\section{ASSETS IN DS}

In order to identify cyber-risks in a DS, assets and their advanced functionalities and communications are studied in this section. As shown in Figure 1, five assets are present in a DS including SCADA system and servers, HMI, intelligent electronic device (IED), merging unit (MU), switches and gateways. The IEC 61850 process bus in red and IEDs are new assets that are integrated for substation digitalization in ECODIS project. The assets' functionalities are listed as follows:

- Server is substation server utilized for managing, securing, and delivering substation information to power utilities control system. The server provides substation operators with the information they need to improve the operability and reliability [GE 2020] as explained below:

- Remote access: allows the specialists to take control of the substation test computer over a secure connection.

- Multi-function software: provides substation with remote access software for data collection, protocol translation, automation logic, and event file collection.

- Event file collection: provides fault record collection and supports secure file transfer to centralized archive. File transfer are encrypted for third party communications [GE 2020].

- SCADA system provides monitoring, supervision, and control of power transmission and distribution in a DS. SCADA utilizes a set of hardware such as servers, gateways, and switches and software for control and monitoring. The monitoring system can create and use a complete system model for automation of power grid [Cherdantseva et al. 2016].

- Remote monitoring: information from substation is displayed for remote monitoring of DS.

- Remote control: equipment control is possible from the substation control center.

- Alarm module: supports analog/digital alarms, configure multiple alarms, and provides alarm summary and logging functions.
- HMI process and visualization system which communicates directly with bay units and protection devices and support IEC 61850 communication standard.

- - Monitoring and control: includes applications with capability of monitoring and control of equipment and substation devices [Csanyi 2017].

- IED is microprocessor-based device connected to substation networks (station bus and process bus) based on IEC 61850 standard that enables communication among components from different vendors in DS. IEDs are building blocks of power system automation and are used for several purposes like protection and metering. IEDs from different vendors provide electricity operators with flexible and menu-driven software programming tools for their settings and configurations [Csanyi 2019]. In DS, most of the remote terminal units (RTU) are replaced with IED due to their several advantages in monitoring and protection as explained below.

- Protection function: SVs are used for real-time processing for protection of DS.

- Metering and power quality analysis: IEDs metering in addition to voltage signals (CT/VT) are used for protection [Bin 2010].

- Self-monitoring and circuit monitoring: IEDs have cardlevel diagnostic capabilities for internal problems. Interface monitoring provides checking the inputs to the IEDs and verifying them by simple methods [Csanyi 2019].

- Event reporting and fault diagnosis: reporting events by utilizing relay IEDs monitoring capabilities.

- Gateway is a communication interface between substation and control center/SCADA allowing SCADA for remote monitoring and controlling in coherence with operations of whole power grid.

- Transmission: gateways are used for transmission of substation indications and measurements to control center/ SCADA, and control center's commands to substation control systems in the grid [GE 2020].

- MU is an interface to connect physical devices (e.g., CT/VT) to IEC 61850-9-2 process bus and IEDs for interoperability of substation devices.

- Digital interface: MU measures the current from transformers, merges and sends them to protective devices in $\mathrm{SV}$, digital format. It is an interface between switchyard devices and protective devices in the DS [GE 2020].

- IEC 61850 converter: MU support event messaging, time synchronization, sending SV over process bus. In DS, SVs are instantaneous values of analogue signals (voltage, current, etc.) that are sampled and digitalized.

\subsection{Communication among Assets}

DS has heterogeneous communication network and environment consist of software, hardware, and applications. Several communication services are present for monitoring and control. Communication among DS assets are based on 3 services: client/server, generic object oriented substation event (GOOSE), and sampled values (SV).

Client/Server: station controller/SCADA/servers communicate with DS components through client/server communication. This 


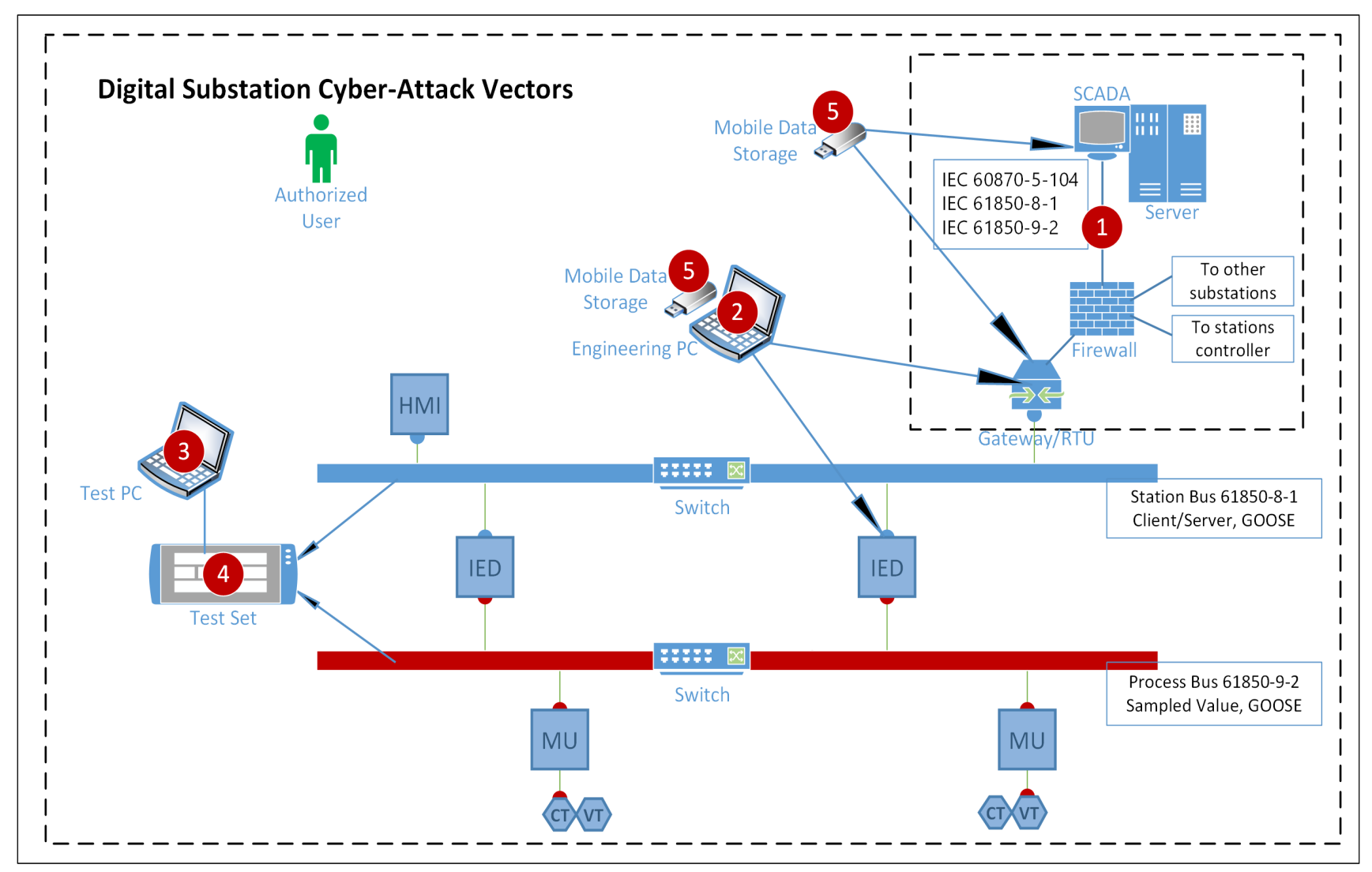

Figure 1: Architecture of a DS including the assets and corresponding cyber-attack vectors. Five potential cyber-attack vectors are identified to affect cyber-security of physical substation and control center/SCADA system.

communication is via point-to-point sessions for central monitoring and control purposes used for sending/receiving commands, reports, $\operatorname{logs}$, and file transfer.

GOOSE: real-time data broadcast for station-wide applications for transferring binary data, indications and commands, communication among IEDs, communication among station controller/ gateway and IEDs along station bus and process bus [Bin 2010].

SV: real-time data broadcast for collecting measurements from process level which are converted measurements from CTs and VTs to digital values by MU, for sending along process bus. The communication among MUs and IEDs are through SVs.

We focus on these assets for discovering cyber-attack vectors. The critical assets are those with highest functionalities and communications with components of DS such as SCADA and IED.

\section{CYBER RISK IDENTIFICATION}

To address cyber-risks in DS, a 3-step methodology is utilized for cyber-risk identification [MITRE 2020b]. Firstly, cyber-attack vectors through DS assets and components are recognized. Secondly, the cyber-attack tactics and their impact on assets are explored Thirdly, mitigation plans are defined to preserve cyber-security in a DS.

\subsection{Cyber-Attack Vectors}

A cyber-attack is an event where an attacker modifies, degrades, or disable at least one component used for protection, automation, or control in ICS. In power grids, if an attacker influence one or more substations it can have severe consequences for the whole grid. Therefore, cyber-attack vectors are required to be recognized, and effective cyber-security measures must be implemented not only in the control centers, but also in substations [Cherdantseva and Hilton 2013]. To address the cyber-attack vectors for ECODIS project, two assumptions are made to study the most relevant cyberattacks and threats that are likely to occur in a DS. First, only authorized users are allowed to enter the substation and only engineers and managers that have permissions can have access to physical devices. Second, remote access to DS is possible through remote access software for remotely control of devices and computers.

Figure 1 depicts cyber-attack vectors on a DS. Four devices may connect to DS assets: mobile data storage, engineering PC, test PC, and test set [Klien 2019]. According to our investigations, DS is prone to cyber-attacks through five vectors listed below:

Path 1: Attack through control center/SCADA. There are cyberthreats of unauthorized remote access, server data collection and modification, and gateway device software manipulation which leads to gain access to command and control the whole substation. 
Table 1: General cyber-attack tactics to affect ICS environments based on MITRE ATT\&CK.

\begin{tabular}{|c|c|c|c|c|c|}
\hline & Tactic & Description & $\mathrm{C}$ & I & A \\
\hline 1 & Collection & gather data of interest and domain knowledge & $\times$ & & \\
\hline 2 & Command and Control & communicate with and control compromised systems, controllers, and platforms & $\times$ & $\times$ & $\times$ \\
\hline 3 & Discovery & figure out ICS environment & $\times$ & & \\
\hline 4 & Evasion & avoid being detected & $x$ & $\times$ & \\
\hline 5 & Execution & run malicious code & $\times$ & $\times$ & $\times$ \\
\hline 6 & Impact & manipulate, interrupt, or destroy ICS systems, data, and surrounding environment & $\times$ & $\times$ & $\times$ \\
\hline 7 & Impair Process Control & manipulate, disable, or damage physical control processes & $\times$ & $\times$ & $\times$ \\
\hline 8 & Inhibit Response Function & $\begin{array}{l}\text { prevent safety, protection, quality assurance, and operator intervention } \\
\text { functions from responding to a failure, hazard, or unsafe state }\end{array}$ & $\times$ & $\times$ & \\
\hline 9 & Initial Access & get into ICS environment & $\times$ & & \\
\hline 10 & Lateral Movement & move through ICS environment & $\times$ & & \\
\hline 11 & Persistence & maintain foothold in ICS environment & $x$ & $\times$ & \\
\hline
\end{tabular}

Path 2: Attack through Engineering PC. A PC may contain malware and by connecting the PC to relay the malware can execute and install itself on IED or SCADA servers. Device settings that are accessible through Engineering PC can be an cyber-attack vector.

Path 3: Attack through Testing PC. A testing PC is connected directly or via Test Set to station bus for testing and there is potential threat to infect substation components IEDs, HMI, and MU through this connection. Test documents could be a potential attack vector while Test PC is connected to station bus or process bus.

Path 4: Attack through Test Set. Testing devices may become entry path by themselves as it is possible for attackers to exploit network's and devices' vulnerabilities.

Path 5: Attack through storage device. There is a threat to execute malicious software or modify IED or SCADA system/software through connecting an infected devices to asset's ports.

It should be noted that the test PC and test devices must be substation hardened [Dolezilek 2000] to meet the DS cyber-security requirements specially while performing remote testing. Based on the cyber-attack vector, physical substation is required to be secured to better protect critical infrastructure and control center/SCADA for secure remote access to the DS.

\subsection{Cyber-Risk Impact}

The cyber-attack vectors may affect cyber-security measures in the DS. Confidentiality, Integrity, and Availability (CIA) [Saltzer and Schroeder 1975] is a well-known model for security policy and effective cyber-security measure development that consists of three concepts:

Confidentiality: to ensure authorized access to sensitive information implemented using security mechanisms such as username/ password, access control models and policies, encryption etc.

Integrity: to ensure the accuracy, consistency and reliability of information to its original purpose and modification of information is only possible for authorized user.

Availability: to ensure authorized access to information and prevent services from getting denied [Saltzer and Schroeder 1975].

According to MITRE ATT\&CK, in an ICS environment 11 cyberattack tactics are effective against security measures, namely, collection, command and control, discovery, evasion, execution, impact, impair process control, in habit response function, initial access, lateral movement, and persistence [MITRE 2020a]. Attackers may use these tactics to influence ICS and affect one or more CIA measures. As shown in Table 1 and based on tactic's definition, collection, discovery, initial access, lateral movement affects confidentiality due to unauthorized access to ICS environment and sensitive information. Evasion, inhabit response function, persistence affect confidentiality and integrity because of unauthorized access to devices and ability to unauthorized modification. Command and control, execution, impact, impair process control are the most influential attack tactics on DS because they may be used to affect all CIA measures and may cause serious damage to assets, denial of service, and manipulation of power system.

We estimated the impact of 11 tactics from Table 1 and corresponding cyber-attack techniques on each asset for the DS. The most relevant tactics and techniques are pointed in Table 2 based on MITRE ATT\&CK definitions. The tactics' threat are assessed in terms of their impact [Bernsmed et al. 2019] as listed below:

- Minor

- Minor or insignificant impact on services for users.

- No interruption of power.

- No damage to equipment.

- Insignificant economic loss.

- Moderate

- Local impact affecting small number of users.

- limited number of affected devices and users loss of power for a limited time.

- Minor damage to equipment.

- small (recoverable) economic loss.

- Major

- Serious consequence in a local community.

- Loss of power for a long period of time for limited users.

- Damage to equipment and/or personnel.

- Major economic loss.

- Critical

- Essential services or critical infrastructure are affected.

- Loss of power in large parts for long period of time.

- Sever damage to equipment and/or loss of human life.

- Irreparable economic loss. 
Table 2: Potential cyber-attack techniques with respect to cyber-attack vectors/entry paths and tactics on a DS based to MITRE ATT\&CK, and their impact scale on corresponding component.

\begin{tabular}{|c|c|c|c|c|}
\hline Assets & Path & Tactic & Cyber-attack technique & Impact \\
\hline $\begin{array}{c}\text { SCADA/ } \\
\text { Servers }\end{array}$ & $1,2,5$ & $\begin{array}{l}\text { Collection } \\
\text { Command and control } \\
\text { Discovery } \\
\text { Execution } \\
\text { Impact } \\
\text { Initial access } \\
\text { Lateral movement }\end{array}$ & $\begin{array}{l}\text { Automated Collection, Data from Information Repositories, } \\
\text { Monitor Process State, Role Identification, Screen Capture } \\
\text { Commonly Used Port, Connection Proxy, Standard Application Layer Protocol } \\
\text { Control Device Identification, Network Sniffing, Remote System Discovery, } \\
\text { I/O Module Discovery } \\
\text { Command-Line Interface, Execution through API, Graphical User Interface, } \\
\text { Man in the Middle, Project File Infection, User Execution } \\
\text { Damage to Property, Denial of Control, Denial of View, Loss of Availability, } \\
\text { Loss of Control, Loss of Safety, Manipulation of Control, } \\
\text { Internet Accessible Devic, External Remote Services } \\
\text { Default Credentials, Exploitation of Remote Services, Remote File Copy } \\
\text { Valid Accounts }\end{array}$ & $\begin{array}{l}\text { Minor } \\
\text { Major } \\
\text { Minor } \\
\text { Critical } \\
\text { Critical } \\
\text { Minor } \\
\text { Moderate }\end{array}$ \\
\hline HMI & $1,3,4$ & $\begin{array}{l}\text { Command and control } \\
\text { Discovery } \\
\text { Impact } \\
\text { Impair process control }\end{array}$ & $\begin{array}{l}\text { Commonly Used Port, Connection Proxy } \\
\text { Control Device Identification, Remote System Discovery } \\
\text { Damage to Property, Denial of Control, Denial of View, Loss of Availability, } \\
\text { Loss of Control } \\
\text { Unauthorized Command Message }\end{array}$ & $\begin{array}{l}\text { Major } \\
\text { Minor } \\
\text { Critical } \\
\text { Major }\end{array}$ \\
\hline IED & $\begin{array}{c}1,2,3 \\
4,5\end{array}$ & $\begin{array}{l}\text { Collection } \\
\text { Discovery } \\
\text { Execution } \\
\text { Impact } \\
\text { Impair process control } \\
\text { Initial access } \\
\text { Lateral movement }\end{array}$ & $\begin{array}{l}\text { Automated Collection, Program Upload, Role Identification } \\
\text { Control Device Identification, Network Connection Enumeration } \\
\text { Network Service Scanning, Network Sniffing, Remote System Discovery, } \\
\text { Command-Line Interface, Execution through API, Man in the Middle, } \\
\text { Project File Infection, User Execution } \\
\text { Damage to Property, Denial of Control, Denial of View, Loss of Availability, } \\
\text { Loss of Control, Manipulation of Control } \\
\text { Modify Control Logic, Modify Parameter, Module Firmware, } \\
\text { Unauthorized Command Message } \\
\text { Internet Accessible Device } \\
\text { Default Credentials, Remote File Copy, Valid Accounts }\end{array}$ & $\begin{array}{l}\text { Minor } \\
\text { Minor } \\
\text { Critical } \\
\text { Critical } \\
\text { Major } \\
\text { Minor } \\
\text { Moderate }\end{array}$ \\
\hline $\mathrm{MU}$ & $2,3,4$ & $\begin{array}{l}\text { Collection } \\
\text { Impact } \\
\text { Impair process control }\end{array}$ & $\begin{array}{l}\text { Detect Operating Mode, Detect Program State, Location Identification } \\
\text { Damage to Property, Loss of View, Manipulation of Control } \\
\text { Modify Parameter }\end{array}$ & $\begin{array}{l}\text { Moderate } \\
\text { Critical } \\
\text { Major }\end{array}$ \\
\hline $\mathrm{CT} / \mathrm{VT}$ & 3,4 & $\begin{array}{l}\text { Collection } \\
\text { Impact } \\
\text { Impair process control }\end{array}$ & $\begin{array}{l}\text { Automated Collection, Detect Operating Mode, Location Identification } \\
\text { Damage to Property } \\
\text { Modify Parameter }\end{array}$ & $\begin{array}{l}\text { Minor } \\
\text { Critical } \\
\text { Major }\end{array}$ \\
\hline
\end{tabular}

In Table 2 the DS assets and corresponding attack-vector/entry path are listed. Based on MITRE ATT\&CK, attacker may apply each tactic by using certain techniques to cause intended effects on assets in DS. We estimated collection, discovery, initial access impact is Minor since the cyber-attack might not cause interruption of power of cause damage to equipment affecting assets through attack techniques automated data collection, monitor process state, role identification, etc. Lateral movement impact is Moderate because of modification of data and possible damage to equipment by affecting assets through attack techniques: default credentials, remote file copy, etc. However, command and control, impair process control are estimated to have Major impact, since they may cause damage and loss of power for limited time. The most severe tactics are impact and execution where the impact is estimated to be Critical due to higher possibility in loss of power and equipment damage that might leads to irreparable economic loss. This damages are possible by denial of control, loss of availability, manipulation of control, and command-line interface, man-in-the-middle as listed in Table 2. We can conclude that SCADA system and servers are the most critical assets of a DS as several cyber-attack techniques are applicable on these assets. They are required to be secured due to vulnerability caused by remote access. In Figure 1 the box around the control center/SCADA shows this critical region.

\subsection{Mitigation Plans}

According to cyber-attack vectors in section 3.1 and cyber-risk impacts in section 3.2, we defined mitigation plans in order to preserve CIA measures. Table 3 presents proposed mitigation plans and strategies for protecting DS against potential cyber-attacks. We defined 6 measures namely Confidentiality of data, Integrity of data, Availability of data, Controllability, Transparency, and Breach communication for DS security policy and effective cyber-security 
Table 3: Mitigation strategies for protection of DS against cyber-attacks.

\begin{tabular}{|c|c|}
\hline Measures & DS Policy \\
\hline $\begin{array}{l}\text { Confidentiality of data: Protect data from } \\
\text { unauthorized disclosure. }\end{array}$ & $\begin{array}{l}\text { - Encryption: Data in DS must be encrypted at rest (i.e., data stored at cloud service } \\
\text { or other data storage environment) and in transit (i.e., data moving around between } \\
\text { system components or between different systems). } \\
\text { - Authorization: Appropriate access control mechanisms (e.g., role-based access con- } \\
\text { trol (RBAC), attribute-based access control (ABAC)) must be implemented to restrict } \\
\text { access to data with full audit trail and reporting logs. } \\
\text { - Authentication: Authorized user in the DS must be authenticated to the system } \\
\text { using a strong password policy, biometrics or multi-factor authentication mechanisms. } \\
\text { - Principle of least privilege: All authorized user must have a least privilege and } \\
\text { minimum required access. } \\
\text { - Secure communication links: Ensure that all communication links in DS are se- } \\
\text { cured with protocols that provide message confidentiality. }\end{array}$ \\
\hline $\begin{array}{l}\text { Integrity of data: Protect data from unautho- } \\
\text { rized intentional modification, user error, soft- } \\
\text { ware and hardware error as well as failures } \\
\text { comes from the act of nature. }\end{array}$ & $\begin{array}{l}\text { - Authorization: DS must be implemented with an appropriate authorization mecha- } \\
\text { nism with least privileges and separation of duties principles. } \\
\text { - Data hashing and signing: All confidential data must in DS be hashed and signed } \\
\text { (digital signature) to ensure that the data is valid (untampered and came from the } \\
\text { correct/expected source) and enforce non-repudiation. } \\
\text { - Secure audit trails: All activities (such as successful and unsuccessful authentication) } \\
\text { and sensitive data must be logged, audit and recorded. } \\
\text { - Cryptographic protocols: Cryptographic protocols such as TLS/SSL must be used } \\
\text { (in case of remote connection via Internet) to ensure a secure (encrypted) communica- } \\
\text { tion between system components. }\end{array}$ \\
\hline $\begin{array}{l}\text { Availability of data: Ensure that information } \\
\text { and system remain available to users when } \\
\text { needed. }\end{array}$ & $\begin{array}{l}\text { - Redundancy and high availability: DS physical components and services must be } \\
\text { replicated to protect all DS services against the failure of single part. } \\
\text { - Fault tolerance: DS physical components and services must be protected against } \\
\text { disruption from a small failure. }\end{array}$ \\
\hline $\begin{array}{l}\text { Controllability: System should provide the au- } \\
\text { thorized user (includes data subjects) with effec- } \\
\text { tive means of control concerning their activities } \\
\text { and data. }\end{array}$ & $\begin{array}{l}\text { - The possibilities regarding consent and data subject rights must be supported by } \\
\text { technological means. }\end{array}$ \\
\hline $\begin{array}{l}\text { Transparency: Ensure that the authorized } \\
\text { users are sufficiently informed about the means } \\
\text { of operation of the systems. }\end{array}$ & $\begin{array}{l}\text { - Transparency: DS users must be provided with all measure to support and enable } \\
\text { electronic access /information/ feedback. }\end{array}$ \\
\hline $\begin{array}{l}\text { Breach communication: DS must be devel- } \\
\text { oped with an effective breach communications } \\
\text { strategy such as be prepared proactively, breach } \\
\text { response plan, accurate and timely notice com- } \\
\text { munications etc. }\end{array}$ & $\begin{array}{l}\text { - Effective Communications Strategy: DS must be prepared proactively for data } \\
\text { breaches by conducting compliance and security assessments; plans and test for breach } \\
\text { response strategies must include the breach communications component and DS must } \\
\text { provide accurate and timely notice communications to the data subject. }\end{array}$ \\
\hline
\end{tabular}

measure development. In order to provide confidentiality, data must be encrypted, appropriate access control mechanisms must be implemented, and users must be authenticated to the system using strong passwords, biometrics or multi-factor authentication. For integrity of data, data must be hashed and signed for data validation, all activities and sensitive data must be logged, and cryptographic protocols must be used for remote access. To ensure availability, DS physical components and services should be replicated to protect DS against failure and disruption of single part or from small failure. DS should provide the authorized users with effective means of control. Authorized users must be informed sufficiently about the means of operations though enabling electronic access/information/feedback. DS must be developed with an effective breach communications strategy such as be prepared proactively, breach response plan, and accurate notice communications.

Additionally, intrusion detection system (IDS) is crucial for monitoring the DS network for malicious activity. Real-time IDS, monitors network traffic, detects, and responds to suspicious event [Liu et al. 2009]. Detection algorithms are important to respond timely and appropriately to abnormal situations. SCADA's data flow, activities, and measurement data from physical devices should be continuously monitored for anomalies. Anomaly detection approaches should be domain-specific for the DS. Normal and dynamic operational states of the DS should be be under observation all the time and firewalls should be present for remote access. 


\section{CONCLUSIONS AND FUTURE WORK}

In this paper, cyber-security risks and their consequences on security measures are analyzed in order to maintain security and reliability for the power grids. A cyber-risk identification methodology is addressed by identifying cyber-attack vectors on a DS, then evaluating the attack's impact on assets based on MITRE ATT\&CK and CIA triad, and consequently, defining mitigation plans. We conclude that trust boundaries should be defined for identified cyber-risks in DS: 1) for physical substation to protect critical infrastructure and 2) for control center/SCADA system to secure remote access. The goal is to recognize and prevent potential cyberattacks to protect power utilities control system. This work is in progress for cyber-risk assessment of DS in ECODIS project in future.

\section{REFERENCES}

Mohamed Abomhara et al. 2015. Cyber security and the internet of things: vulnerabilities, threats, intruders and attacks. Journal of Cyber Security and Mobility 4, 1 (2015), 65-88.

Mohamed Abomhara and Geir M Køien. 2014. Security and privacy in the Internet of Things: Current status and open issues. In 2014 international conference on privacy and security in mobile systems (PRISMS). IEEE, 1-8.

Drew Baigent, Mark Adamiak, Ralph Mackiewicz, and G. M. G. M. Sisco. 2004. IEC 61850 communication networks and systems in substations: An overview for users. SISCO Systems (2004).

Karin Bernsmed, Martin Gilje Jaatun, and Christian Frøystad. 2019. Is a Smarter Grid Also Riskier? In International Workshop on Security and Trust Management, 36-52.

Wang Bin. 2010. Substation automation solution with IEC61850. ABB Group (2010).

Yulia Cherdantseva, Pete Burnap, Andrew Blyth, Peter Eden, Kevin Jones, Hugh Soulsby, and Kristan Stoddart. 2016. A review of cyber security risk assessment methods for SCADA systems. Computers \& security 56 (2016), 1-27.
Yulia Cherdantseva and Jeremy Hilton. 2013. A reference model of information assurance \& security. International Conference on Availability, Reliability and Security.

Edvard Csanyi. 2017. Four design criteria for human machine interface (HMI) in a substation. Electrical Engineering Portal (2017).

Edvard Csanyi. 2019. IED (Intelligent Electronic Device) advanced functions that make our life better. Electrical Engineering Portal (2019).

David J Dolezilek. 2000. Choosing between communications processors, RTUS, and PLCS as substation automation controllers. Schweitzer Engineering Laboratories, Inc. white paper (2000).

ECODIS. 2019. Engineering and Condition Monitoring of Digital Substations (ECODIS). Retrieved May 10, 2020 from https://www.sintef.no/en/projects/ecodis/

GE. 2020. GE grid solutions. Retrieved May 10, 2020 from https://www.gegridsolutions. $\mathrm{com} /$

Andreas Klien. 2019. New approach for detecting cyber intrusions in IEC 61850 substations. PAC World (2019).

William Knowles, Daniel Prince, David Hutchison, Jules Ferdinand Pagna Disso, and Kevin Jones. 2015. A survey of cyber security management in industrial control systems. International journal of critical infrastructure protection 9 (2015), 52-80.

Chen-Ching Liu, Chee-Wooi Ten, and Manimaran Govindarasu. 2009. Cybersecurity of SCADA systems: Vulnerability assessment and mitigation. In 2009 IEEE/PES Power Systems Conference and Exposition.

Rannveig SJ Loken, Nargis Hurzuk, Linda Stensrud, Bendik Ohrn, and et al. 2018. Experience with process bus in Statnett R\&D project Digital substation. CIGRE (2018).

MITRE. 2020a. ATT\&CK for Industrial Control Systems. Retrieved May 10, 2020 from https://collaborate.mitre.org/attackics/index.php/Main Page

MITRE. 2020b. Systems Engineering Guide: Risk Management. Retrieved May 10, 2020 from https://bit.ly/3byYPAA

Naiara Moreira, Elias Molina, Jesus Lazaro, and Eduardo Jacoband Armando Astarloa. 2016. Cyber-security in substation automation systems. Renewable and Sustainable Energy Reviews 54 (2016), 1552-1562.

Jerome H Saltzer and Michael D Schroeder. 1975. The protection of information in computer systems. In Proceedings of the IEEE, 1278-1308.

Linda Stensrud, Bendik Ohrn, Rannveig SJ Loken, Nargis Hurzuk, and Alex Apostolov. 2018. Testing of Intelligent Electronic Device (IED) in a digital substation. The fournal of Engineering 15 (2018), 900-3.

Keith Stouffer, Joe Falco, and Karen Scarfone. 2011. Guide to industrial control systems (ICS) security. NIST special publication 800 (2011). 